\title{
Exploring Natural Alternatives for Annual Bluegrass Control
}

\author{
Jacob Taylor ${ }^{1, * \mathbb{D}}$, Lambert McCarty ${ }^{1}$ and Robert Kerr ${ }^{2}$ \\ 1 Plant and Environmental Science Department, Clemson University, Clemson, SC 29634, USA; \\ bmccrty@clemson.edu \\ 2 Bayer Environmental Sciences, Clayton, NC 27513, USA; robert.kerr@bayer.com \\ * Correspondence: jwtaylo@g.clemson.edu
}

Citation: Taylor, J.; McCarty, L.; Kerr, R. Exploring Natural Alternatives for Annual Bluegrass Control. Horticulturae 2021, 7, 365. https:// doi.org/10.3390/horticulturae7100365

Academic Editor: Douglas

D. Archbold

Received: 4 August 2021

Accepted: 29 September 2021

Published: 5 October 2021

Publisher's Note: MDPI stays neutral with regard to jurisdictional claims in published maps and institutional affiliations.

Copyright: (c) 2021 by the authors. Licensee MDPI, Basel, Switzerland. This article is an open access article distributed under the terms and conditions of the Creative Commons Attribution (CC BY) license (https:/ / creativecommons.org/licenses/by/ $4.0 /)$.

\begin{abstract}
A field experiment was conducted to identify and examine the effectiveness of potential non-traditional chemical products and alternatives for controlling annual bluegrass (Poa annua L.), a plant with increasing herbicide resistant problems. In managed turf systems annual bluegrass can be a difficult winter annual weed to control having negative impacts on turfgrass quality. This study included 12 different treatments [untreated check, baking soda, white vinegar + lemon juice, Suppress herbicide, superphosphate (0-20-0), clove oil, Weed Zap, Avenger Weed Killer, Fiesta Turf Weed Killer, Ecologic Weed \& Grass Killer, Alcohol (43\% ethanol), and Pool Time Algicide] applied to a TifEagle bermudagrass (Cynodon dactylon $\times$ C. traansvalensis) putting green where a natural infestation of annual bluegrass was present. Treatments were assessed visually for annual bluegrass control and turf phytotoxicity 1, 2, and 4 weeks after application. Overall, no treatment provided long-term control which was non-selective. Control was short-lived with annual bluegrass recovery beginning approximately two weeks after applying treatments. Greatest Poa burndown ( 85\%) was temporarily (1 to 2 weeks after application) with a combination of caprylic and capric acids (Suppress Herbicide) and a combination of clove oil and dishwashing detergent but plants fully recovered by three weeks after application. These products also produced similar temporary turf phytotoxicity. Bermudagrass turf phytotoxicity from selective treatments was most evident one week after applying treatments and turf had mostly fully recovered by four weeks after treatment. Suitable alternatives were not identified from products tested.
\end{abstract}

Keywords: alternative weed control; herbicide resistance; non-synthetic products; phytotoxicity

\section{Introduction}

Annual bluegrass (Poa annua L.) is the most troublesome winter annual weed in managed turf systems and has grown to epidemic proportions [1,2]. Having a light green color, it negatively impacts turfgrass quality through prolific seedhead production, clumped growth habit, and lack of stress tolerance [3,4]. Controlling annual bluegrass can be difficult as it exhibits high levels of genetic diversity, rapidly adapts to different climates as well as practices, and has developed widespread herbicide resistance.

Current management programs rely heavily on herbicides for annual bluegrass control even though few truly effective options are available [5]. Frequent herbicide use with the same mode-of-action without implementation of other non-chemical management practices can lead to resistant species with resistance to at least nine different herbicide modes of action $[3,6]$. Therefore, alternative options for control are needed to reduce dependency of synthetic herbicides and to combat herbicide resistance.

Alternative non-chemical products are available to annual bluegrass control, but the effectiveness of these products has not been adequately tested in different turf systems and environments. There have also been untested claims about household compounds or homemade products being used to control weeds. Typically, natural or biological control options are non-selective with all treated plants (desired or not) sustaining damage [7]. The objectives of this study were to further investigate different non-chemical commercial 
products and household compounds for their effectiveness to control annual bluegrass and turfgrass tolerance.

\section{Materials and Methods}

An experiment was conducted at Clemson University, Clemson, S.C., United States in the spring of 2020 (Year 1) and 2021 (Year 2) to identify and evaluate the effectiveness of potential non-chemical products and alternatives for controlling annual bluegrass. A TifEagle bermudagrass (Cynodon dactylon $\times$ C. traansvalensis) putting green (Figure 1) was chosen as the experiment site due to sufficient natural infestation of annual bluegrass present. This study included 12 different treatments consisting of common household compounds (Table 1).

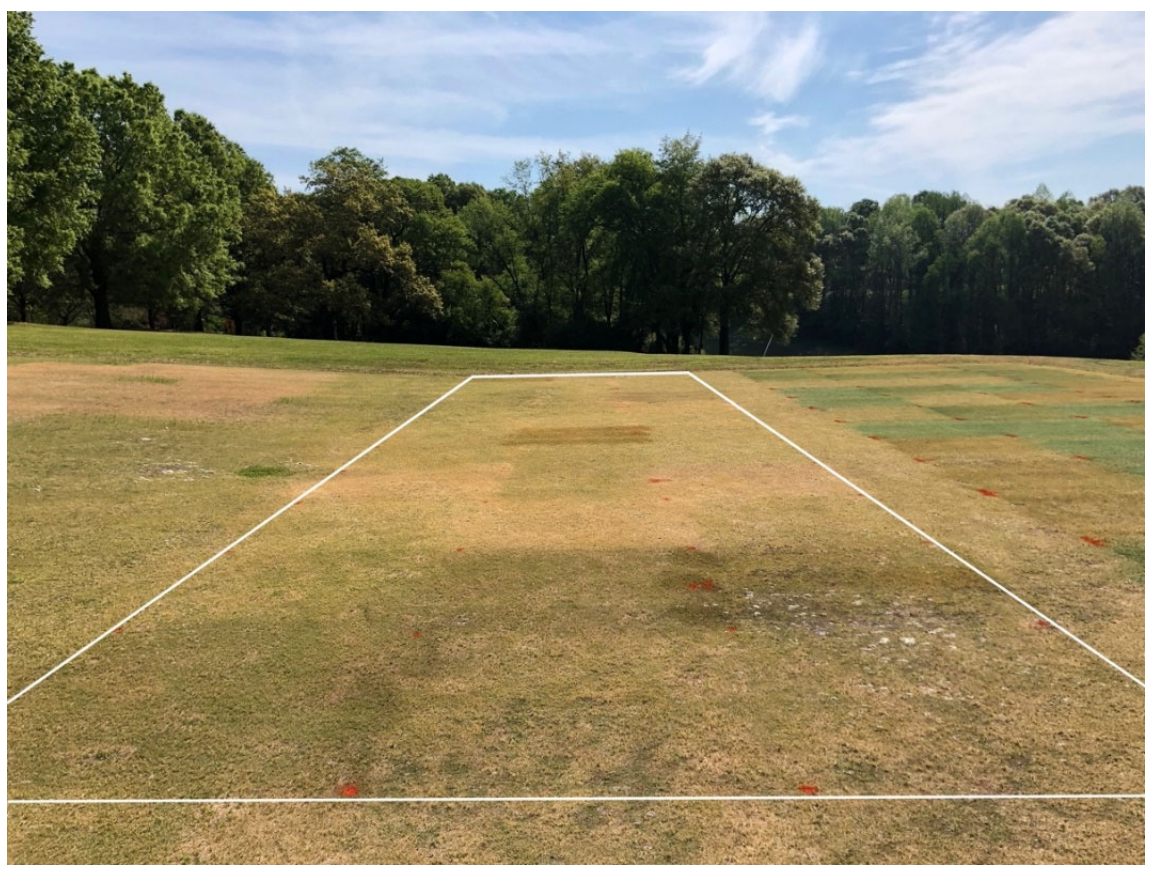

Figure 1. Study plots on TifEagle bermudagrass putting green taken one week after treatment applications.

Table 1. Treatments using common products for postemergence Poa annua control.

\begin{tabular}{|c|c|c|}
\hline & Treatment/Product & Rate $^{+}$ \\
\hline 1 & Untreated Check & \\
\hline 2 & Baking Soda + Dawn Dishwashing Soap * & $269 \mathrm{~kg} / \mathrm{ha}(240 \mathrm{lb} / \mathrm{a})$ \\
\hline 3 & White Vinegar + Lemon Juice + Dawn & $234 \mathrm{~L} / \mathrm{ha}(25 \mathrm{gal} / \mathrm{a})$ \\
\hline 4 & Suppress Herbicide ( $47 \%$ caprylic acid $+32 \%$ capric acid) & $35 \mathrm{~L} / \mathrm{ha}(480 \mathrm{fl} \mathrm{oz} / \mathrm{a})$ \\
\hline 5 & Superphosphate (0-20-0) + Dawn & $122 \mathrm{~kg} / \mathrm{ha}(108.9 \mathrm{lb} / \mathrm{a})$ \\
\hline 6 & Clove Oil + Dawn & $26 \mathrm{~L} / \mathrm{ha}(384 \mathrm{fl} \mathrm{oz} / \mathrm{a})$ \\
\hline 7 & Weed Zap ( $45 \%$ clove oil $+45 \%$ cinnamon oil) & $26 \mathrm{~L} / \mathrm{ha}(384 \mathrm{fl} \mathrm{oz} / \mathrm{a})$ \\
\hline 8 & Avenger Weed Killer (70\% d-limonene (citrus oil)) & $83 \mathrm{~L} / \mathrm{ha}(1140 \mathrm{fl} \mathrm{oz} / \mathrm{a})$ \\
\hline 9 & Fiesta Turf Weed Killer (26.5\% FeHEDTA) & $22 \mathrm{~L} / \mathrm{ha}(300 \mathrm{fl} \mathrm{oz} / \mathrm{a})$ \\
\hline 10 & Ecologic Weed \& Grass Killer (0.5\% cinnamon oil) & $26 \mathrm{~L} / \mathrm{ha}(384 \mathrm{fl} \mathrm{oz} / \mathrm{a})$ \\
\hline 11 & Alcohol $(43 \%$ ethanol $)+$ Dawn & $31 \mathrm{~L} / \mathrm{ha}(420 \mathrm{fl} \mathrm{oz} /)$ \\
\hline 12 & Pool Time Algicide (50\% ADBAC concentrate) + Dawn & $0.5 \% v / v$ \\
\hline
\end{tabular}

${ }^{+}$Spray volume $=561 \mathrm{~L} / \mathrm{ha}(60 \mathrm{gal} / \mathrm{ac}) .{ }^{*}$ Dawn dishwashing soap applied at $0.5 \% v / v$ (volume/volume). 
Treatments were applied on 30 March 2020, and 2021 using a carbon dioxide $\left(\mathrm{CO}_{2}\right)$ pressurized backpack sprayer delivering $60 \mathrm{gal} \mathrm{ac}^{-1}\left(561 \mathrm{~L} \mathrm{ha}^{-1}\right)$. Applications were made during spring greenup, typically when bermudagrass is most sensitive to herbicide applications. Plots measured $4.9 \times 4.9$ feet $(1.5 \times 1.5 \mathrm{~m})$ and were arranged in a randomized complete block design with three replications in each trial. The site was maintained to normal putting green standards at a mowing height of $0.125 \mathrm{inch}(3.2 \mathrm{~mm})$ and irrigation provided as needed to prevent wilting. Plots were not mowed during the study to better display results from treatments.

Treatments were assessed visually for annual bluegrass control and turf phytotoxicity 1,2 , and 4 weeks after application. Visual control of annual bluegrass was rated on a scale of $0 \%$ to $100 \%$, where $0 \%=$ no control and $100 \%=$ complete control. Phytotoxicity on TifEagle bermudagrass was also rated visually on a scale of $0 \%$ to $100 \%$, where $0 \%=$ no phytotoxicity, $30 \%$ = maximum acceptable damage level, $100 \%=$ complete turfgrass death

Statistical analyses were performed using analysis of variance (ANOVA) within JMP ${ }^{\circledR}$ Pro system (Version 13.2.0, SAS Institute, Cary, NC 27513, USA). Means separation was analyzed using a Student's $t$ test at $\alpha \geq 0.05$.

\section{Results}

Significant differences occurred between evaluation dates and years; therefore, results are presented separately by evaluation date and year. Annual bluegrass control from treatments were mostly non-selective and visually appeared to burndown everything, including the desirable turf (Table 2). However, results were relatively short-lived with most products providing little to no long-term control.

Table 2. Visual control of annual bluegrass from non-synthetic treatments. Different letters within a column indicate statistical differences.

\begin{tabular}{|c|c|c|c|c|c|c|}
\hline \multirow{3}{*}{ Treatments } & \multicolumn{6}{|c|}{$\%$ Poa annua Control } \\
\hline & \multicolumn{3}{|c|}{ Year 1} & \multicolumn{3}{|c|}{ Year 2} \\
\hline & $1 \mathrm{WAT}^{*}$ & $2 \mathrm{WAT}^{*}$ & $4 \mathrm{WAT}^{*}$ & $1 \mathrm{WAT}^{*}$ & $2 \mathrm{WAT}^{*}$ & 4 WAT * \\
\hline Untreated Check & $0 c$ & $0 \mathrm{~b}$ & Ons & $0 g$ & $0 \mathrm{e}$ & Ons \\
\hline Baking Soda + Dawn Dishwashing Soap & $6.7 \mathrm{bc}$ & $3.3 \mathrm{~b}$ & 3.3 & $0 \mathrm{~g}$ & $0 \mathrm{e}$ & Ons \\
\hline White Vinegar, Lemon Juice, Dawn & $3.3 \mathrm{bc}$ & $3.3 b$ & 0 & $43.3 \mathrm{cde}$ & 10.0de & Ons \\
\hline Suppress Herbicide ( $47 \%$ caprylic acid $+32 \%$ capric acid) & $50.0 \mathrm{a}$ & $23.3 \mathrm{a}$ & 3.3 & $86.7 \mathrm{a}$ & $66.7 \mathrm{a}$ & Ons \\
\hline Superphosphate (0-20-0) + Dawn & $3.3 \mathrm{bc}$ & $0 \mathrm{~b}$ & 0 & 10.0efg & $0 \mathrm{e}$ & Ons \\
\hline Clove Oil + Dawn & $0 \mathrm{c}$ & $0 \mathrm{~b}$ & 0 & $80.0 \mathrm{ab}$ & $53.3 \mathrm{ab}$ & Ons \\
\hline Weed Zap ( $45 \%$ clove oil + 45\% cinnamon oil) & $10.0 \mathrm{bc}$ & $6.7 \mathrm{~b}$ & 3.3 & 73.3abc & $36.7 \mathrm{bc}$ & Ons \\
\hline Avenger Weed Killer (70\% d-limonene (citrus oil)) & $20.0 b$ & $10.0 \mathrm{~b}$ & 0 & 56.7abcd & $26.7 \mathrm{~cd}$ & Ons \\
\hline Fiesta Turf Weed Killer (26.5\% FeHEDTA) & $16.7 \mathrm{bc}$ & $6.7 \mathrm{~b}$ & 0 & 13.3efg & $3.3 \mathrm{e}$ & Ons \\
\hline Ecologic Weed \& Grass Killer (0.5\% cinnamon oil) & $3.3 \mathrm{bc}$ & $0 \mathrm{~b}$ & 3.3 & $53.3 \mathrm{bcd}$ & 13.3de & Ons \\
\hline Alcohol (43\% ethanol) + Dawn & 0c & $0 \mathrm{~b}$ & 0 & 36.7def & 10.0de & Ons \\
\hline Pool Time Algicide (50\% ADBAC concentrate) + Dawn & $0 \mathrm{c}$ & $0 \mathrm{~b}$ & 0 & $0 \mathrm{~g}$ & $0 \mathrm{e}$ & Ons \\
\hline LSD (0.05) & 18.6 & 11.6 & 5.6 & 32.8 & 19.5 & 0 \\
\hline
\end{tabular}

During Year 1, only plots treated with Suppress Herbicide (caprylic acid + capric acid) and Avenger (citrus oil) had visual control ratings statistically higher than the untreated check. Annual bluegrass control was most noticeable one week after treatment (WAT) with Suppress Herbicide provided best control at 50\% followed by Avenger at $20 \%$ (Figure 2). Results began to wane the second WAT with significant control only observed with Suppress Herbicide (23\%). Annual bluegrass plants continued to recover and by the fourth WAT, no statistical control differences were evident between treatments and untreated check. 


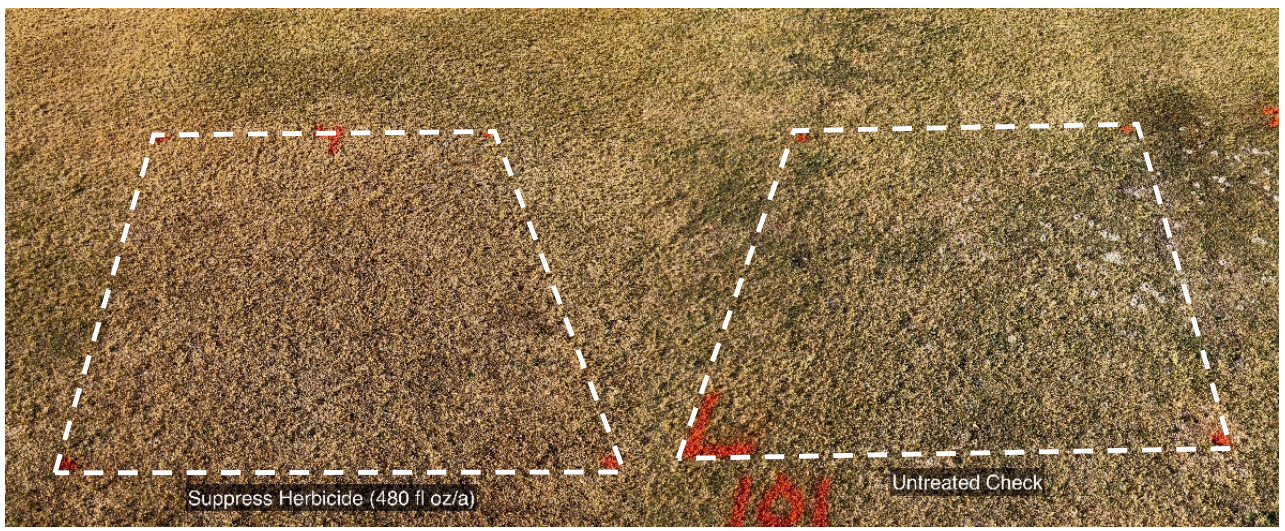

Figure 2. Suppress herbicide (480 fl oz/a) and untreated check one week after treatment (WAT) (Year 1).

In year 2, seven treatments had visual control ratings statistically different than the untreated check. Similar to year 1, annual bluegrass control was most noticeable 1WAT. Suppress Herbicide, Clove Oil + dishwashing soap, Weed Zap (clove oil + cinnamon oil) and Avenger Weed Killer providing best control at between $~ 57$ and $87 \%$ (Table 2). By the second WAT, results began to wane with significant control only observed with Suppress Herbicide ( 67\%), Clove Oil + Dawn (53\%), Weed Zap (37\%), and Avenger Weed Killer $(27 \%)$. Recovery from all treatments was observed by 4 WAT at which time, no statistical differences occurred between treatments and untreated check.

Bermudagrass turf phytotoxicity was observed following the application of selective treatments during both years (Table 3). Phytotoxicity was most evident 1WAT in plots treated with Suppress Herbicide and baking soda plus dishwashing soap but, phytotoxicity was only observed in plots treated with Suppress Herbicide 2WAT during Year 1 (Table 3). No other treatment caused phytotoxicity above the acceptable level at this point in Year 1. The turf had mostly fully recovered by 4WAT.

Table 3. Phytotoxicity of TifEagle bermudagrass from non-synthetic annual bluegrass control treatments. Different letters within a column indicate statistical differences.

\begin{tabular}{|c|c|c|c|c|c|c|}
\hline \multirow{3}{*}{ Treatments } & \multicolumn{6}{|c|}{ \% Bermudagrass Phytotoxicity } \\
\hline & \multicolumn{3}{|c|}{ Year 1} & \multicolumn{3}{|c|}{ Year 2} \\
\hline & 1 WAT * & 2 WAT * & 4 WAT * $^{*}$ & $1 \mathrm{WAT}^{*}$ & 2 WAT * & 4 WAT * \\
\hline Untreated Check & $3.3 \mathrm{bc}$ & $0 \mathrm{~b}$ & $0 \mathrm{~b}$ & $0 \mathrm{e}$ & $\mathrm{Ob}$ & Ons \\
\hline Baking Soda + Dawn Dishwashing Soap & $50.0 \mathrm{a}$ & $13.3 \mathrm{~b}$ & $6.7 \mathrm{ab}$ & $0 \mathrm{e}$ & $0 \mathrm{~b}$ & 0ns \\
\hline White Vinegar, Lemon Juice, Dawn & $3.3 \mathrm{bc}$ & $10.0 \mathrm{~b}$ & Ob & $40.0 \mathrm{bcd}$ & $6.7 \mathrm{~b}$ & Ons \\
\hline Suppress Herbicide ( $47 \%$ caprylic acid $+32 \%$ capric acid) & $50.0 \mathrm{a}$ & $46.7 \mathrm{a}$ & $10.0 \mathrm{a}$ & $86.7 \mathrm{a}$ & $73.3 \mathrm{a}$ & Ons \\
\hline Superphosphate (0-20-0) + Dawn & $0 \mathrm{c}$ & $0 \mathrm{~b}$ & $0 \mathrm{~b}$ & 6.7de & $0 \mathrm{~b}$ & Ons \\
\hline Clove Oil + Dawn & $0 \mathrm{c}$ & $0 \mathrm{~b}$ & $0 \mathrm{~b}$ & $83.3 \mathrm{a}$ & $56.7 \mathrm{a}$ & 0ns \\
\hline Weed Zap ( $45 \%$ clove oil + $45 \%$ cinnamon oil) & $0 \mathrm{c}$ & $3.3 b$ & $3.3 \mathrm{ab}$ & 73.3ab & $23.3 \mathrm{~b}$ & Ons \\
\hline Avenger Weed Killer (70\% d-limonene (citrus oil)) & $16.7 \mathrm{~b}$ & $10.0 \mathrm{~b}$ & $0 \mathrm{~b}$ & $53.3 \mathrm{abc}$ & $23.3 b$ & Ons \\
\hline Fiesta Turf Weed Killer (26.5\% FeHEDTA) & $6.7 \mathrm{bc}$ & $6.7 \mathrm{~b}$ & $0 \mathrm{~b}$ & 10.0de & $0 \mathrm{~b}$ & Ons \\
\hline Ecologic Weed \& Grass Killer (0.5\% cinnamon oil) & $6.7 \mathrm{bc}$ & $6.7 \mathrm{~b}$ & $6.7 \mathrm{ab}$ & $53.3 \mathrm{abc}$ & $23.3 b$ & Ons \\
\hline Alcohol (43\% ethanol) + Dawn & $0 \mathrm{c}$ & $0 \mathrm{~b}$ & $0 \mathrm{~b}$ & $36.7 \mathrm{~cd}$ & $13.3 \mathrm{~b}$ & Ons \\
\hline Pool Time Algicide (50\% ADBAC concentrate) + Dawn & $0 c$ & $0 \mathrm{~b}$ & $0 \mathrm{~b}$ & $0 \mathrm{e}$ & $0 \mathrm{~b}$ & Ons \\
\hline LSD (0.05) & 13.5 & 15.1 & 6.9 & 33.8 & 23.5 & 0 \\
\hline
\end{tabular}

* WAT, weeks after treatment. ns = nonsignificant. 
During Year 2, phytotoxicity ranged from $~ 40$ to $85 \%$ 1WAT in plots treated with Suppress Herbicide, Weed Zap, Avenger, Ecologic Weed \& Grass Killer (cinnamon oil), white vinegar, lemon juice, and ethanol (Table 3). By 2WAT, phytotoxicity above the $30 \%$ maximum level was only observed with Suppress Herbicide (73\%) and Clove Oil + Dawn (57\%). Turf fully recovered by the fourth WAT with no phytotoxicity from treatments observed.

\section{Discussion and Conclusions}

From this study, several non-synthetic chemical products tested could potentially provide some short-term annual bluegrass burndown. However, results were intermediate at best with little to no long-term control from most products evaluated, similar to the low weed control efficacy observed after applying non-chemical products in other turfgrass systems [8]. None of the products tested appear to provide acceptable commercial control with only a single application for this winter annual weed. Control from these products could possibly be improved through multiple applications as some tested product labels mention repeat applications may be necessary for effective control [9,10]. Additional research is needed to determine whether multiple applications of these products can effectively control annual bluegrass without injuring the desired turf.

Certain products also caused short-term (1 to 2 weeks) undesirable turf phytotoxicity. Other research has found similar, non-chemical products have caused injury to desired turf as well $[8,11]$. Similar visual burndown is often observed after applying non-selective herbicides such as glyphosate, diquat, or glufosinate [3]. It is not recommended to apply the products utilized in this research for annual bluegrass control as none are registered herbicides and turf safety has not yet been adequately tested.

Additional research is needed to further evaluate these products for turf safety. Future research may also investigate application timing and optimal rate of such products for optimal weed control. Identifying a non-synthetic chemical product effective for annual bluegrass control that does not damage the desired turf would give a much-needed alternative to reduce synthetic herbicides use.

Technical Contribution No. 7004 of the Clemson University Experiment Station. This material is based upon work supported by NIFA/USDA, under project number SC-1010410.

Author Contributions: Conceptualization, methodology, resources, writing-review and editing, L.M.; validation, formal analysis, data curation, writing—original draft preparation, J.T.; investigation, data curation, R.K. All authors have read and agreed to the published version of the manuscript.

Funding: This research was funded byClemson University Experiment Station.

Conflicts of Interest: The authors declare no conflict of interest.

\section{References}

1. Cross, R.B.; McCarty, L.B.; Tharayil, N.; McElroy, J.S. A Pro 106 to Ala substitution is associated with resistance to glyphosate in annual bluegrass (Poa annua). Weed Sci. 2015, 63, 613-622. [CrossRef]

2. Van Wychen, L. Survey of the Most Common and Troublesome Weeds in the United States and Canada. Weed Science Society of America National Weed Survey Dataset. 2016. Available online: http://wssa.net/wp-content/uploads/2015-Weed-Survey_final/xlsx (accessed on 1 August 2021).

3. McCarty, L.B. Golf Turf Management; CRC Press: Boca Raton, FL, USA, 2018.

4. McCarty, L.B.; Hall, D.W. Common Weeds and Wildflowers; Clemson Univ. Public Service Publishing: Clemson, SC, USA, 2018.

5. Duke, S.O. Why have no new herbicide modes of action appeared in recent years? Pest Manag. Sci. 2012, 68, 505-512. [CrossRef] [PubMed]

6. Heap, I. The International Survey of Herbicide Resistant Weeds. 2018. Available online: www.weedscience.org (accessed on 4 May 2020).

7. Kerr, R.A.; McCarty, L.B.; Harris, J.; McElroy, S. Alternative Control Options for Annual Bluegrass (Poa annua L.). In Proceedings of the ASA/CSSA/SSSA International Annual Meeting, San Antonia, TX, USA, 10-13 November 2019.

8. Patton, A.J.; Braun, R.C.; Weisenberger, D.V. Single applications of natural postemergence weed control options do not provide effective ground ivy control. Crop. Forage Turfgrass Manag. 2019, 5, 1-7. [CrossRef]

9. Anonymous. Avenger Weed Killer Concentrate Product Label; Cutting Edge Formulations, Inc.: Buford, GA, USA, 2010.

10. Anonymous. Suppress Herbicide EC Product Label; Westbridge Agricultural Products: Vista, CA, USA, 2011.

11. Carroll, D.E.; Kaminski, J.E.; Borger, J.A. Efficacy of natural herbicides on dandelion (Taraxacum officinale G.H. Weber ex Wiggers) and white clover (Trifolium repens L.) populations. Int. Turfgrass Soc. Res. J. 2020, 1-11. [CrossRef] 